\title{
Some notes on the Inka constellations
}

\author{
Elluz Pacheco ${ }^{1}$, Soraya Flores ${ }^{1}$ and Erwin Salazar ${ }^{2}$ \\ ${ }^{1}$ Universidad Nacional San Antonio Abad del Cusco, \\ Av. de la Cultura, No. 733, Cusco 51084, Perú \\ email: elita_p_c@hotmail.com soryta18@hotmail.coma \\ ${ }^{2}$ Planetarium Cusco, \\ Reserva Ecológica Turística de Llaullipata, \\ Carretera a Sacsayhuamán s/n, Cusco, Perú \\ email: ersalgar@gmail.com
}

\begin{abstract}
The research carried out so far on the identification of constellations in the Inka civilisation is far from exhaustive, as there is both a great difficulty in obtaining the data and much heterogeneity in the information gathered. A brief presentation is given here.
\end{abstract}

Keywords. Constellations, Inka civilisation, Milky Way

\section{Introduction}

The knowledge of Inka constellations which are still used today in the Andes has undergone a lucky series of 'mutations' and changes brought about by a strong syncretism and in spite of the systematic neglect of the details behind it. They survive in the form of legends and myths inherited from ancestors. Each town or each informant 'interpreted' in its location the names and significance of celestial objects. It is important to emphasize that within the astronomy of the Inkas there are two types of constellations: (a) Stellar constellations or bright stars, made up of individual bright stars which constitute a 'constellation' and grouped together very much as in the greco-roman tradition of the night sky. (b) Dark (or black) constellations, which are condensations of interstellar gas and dust which appear as dark spots or silhouettes against the bright and diffuse the Milky Way. It is well possible that he Inca culture was the only one with these two simultaneous types of constellations on the sky. We can outline the approximate correspondance of the constellations recovered to date, although much work remains to be done. We also briefly discuss two of these constellations and their interpretation within the context of the astronomy in the Inka culture.

\section{Lluthu: a partridge in the coalsack}

Within the so-called "Dark Constellations" of the Incas one stands out for being the darkest and best represented : the bird that symbolises the Andean partridge or Lluthu, also called Chaqwa in other regions, or P'isaqa, Yuktuq, etc. Lluthu is located in the area occupied by the Southern Cross, to the left of Alpha Crucis and constitutes what is currently called the Coalsack Dark Nebula. The shape of the "bag" seen in a cold clear night without Moon and away from city lights is impressive: it is a round spot with a small extension to the South that makes it easy to find. The little bird seems to look towards the southern Celestial Pole. The constellation appears in the morning sky in October and stays until July, i.e., until the end of the harvest of the tubers. 


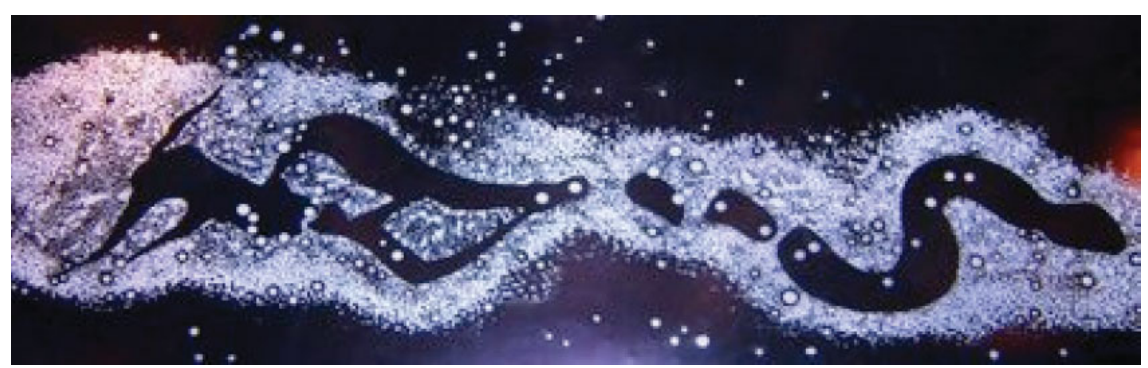

Figure 1. Schematic guide to some dark constellations in the Inka culture. (c) James Arévalo.

Table 1. Some constellations of the Inkas. Note that the location of the Inkas' constellations within the modern constellations does not contain or includes the same stars. Zones are only for reference and asterisms/groups overlap partially only. Originally published at http://qoyllur.blogspot.com/2009/06/constelaciones-inkas-un-listado.html

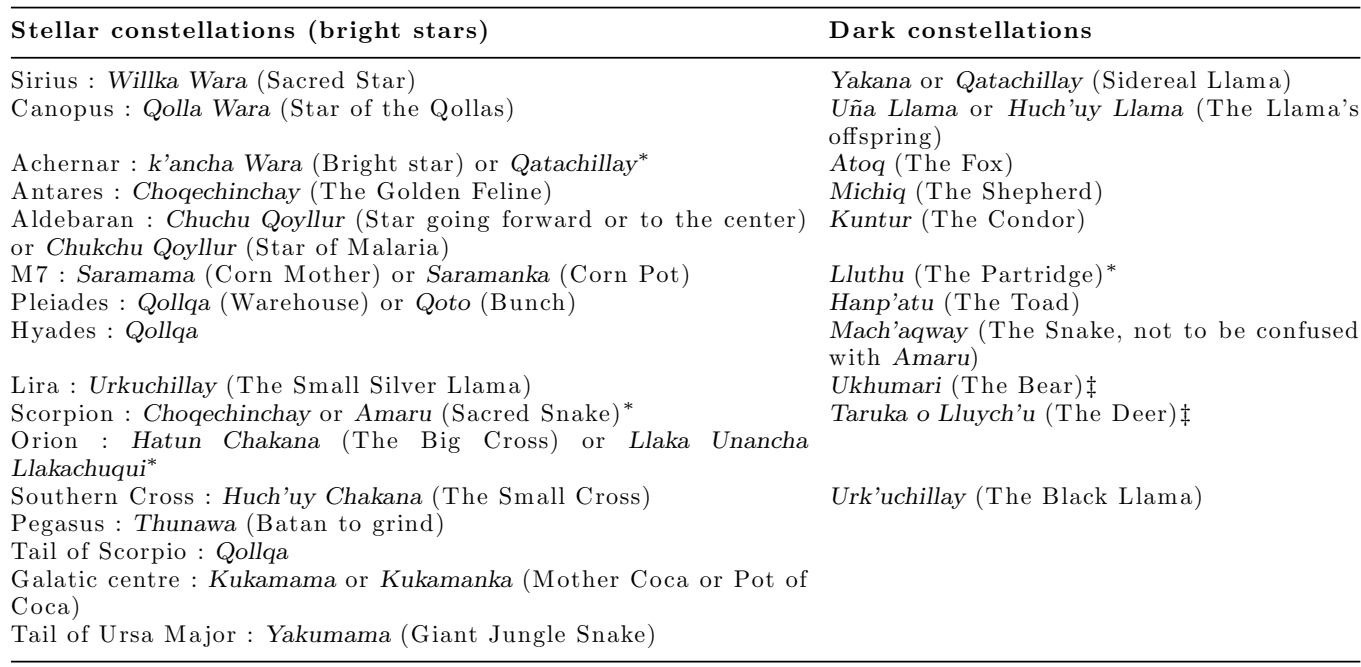

* There are two possible interpretations.

$\ddagger$ Location uncertain.

\section{Willkawara: Sirius the sacred star}

Sirius (Alpha Canis Majoris) is the brightest star in the sky. In the Andes - particularly in Cusco- it is almost exactly at the zenith at nightfall in the days near the Autumn equinox (March 21). We know from ethnographic sources that in some villages the myth of the 'Sacred Star' or Willkawara (from Sacred = Willka, Star = Wara) is still remembered. The myth states that Willkawara is a star that appears at dusk with the rains of December and comes to protect the crops of Andean farmers throughout the maturation period of the potatoes to be harvested and stored. A curious fact on the presence of Willkawara in the Cusco sky is that before Sirius reaches the zenith towards the end of February it is a sign to pick and harvest the first potatoes that belong to the early sowing or maway, whereas when Willkawara happens to be located in the western sky, after the peak in March (a few weeks after the equinox), the expected harvest is largest.

\section{References}

Urton, G. 1981, At the crossroads of the Earth and the Sky (Austin: University of Texas Press) 